\title{
Genetic Control of Fruit Quality, and Prospects for Nutrient Modification
}

\author{
James J. Giovannoni \\ USDA-ARS Plant Soil and Nutrition Laboratory, Tower Road, Ithaca, NY 14853
}

\begin{abstract}
The nutritional challenges in developed nations such as the United States are unique and significant. Minimal nutritional requirements are often not met while caloric requirements are increasingly exceeded. This dichotomy typically results from consumption of excessive snack and processed foods in place of fruits and vegetableswhich are typically the highest sources of fiber and many essential vitamins and nutrients. Advanced plant breeding and genetic enhancement (biotechnology) afford opportunities to address this important health challenge by: 1) increasing the quality of produce (flavor, aroma, appearance, texture) to enhance desirability, thus making fruits and vegetables more competitive with processed foods; and 2) optimizing concentration and bioavailability of essential nutrients to yield crops with greater "health impact." In addition to the United States and developed countries, products developed from said technologies will be important for meeting nutritional needs in underdeveloped and developing nations. Indeed, the best argument for responsible use of genetic engineering will be development of crop plants that enhance the sustainability and quality of human life. Additional benefits will be the impact of health-based added value to the agricultural economy and logical extension of scientific findings to improvement of livestock and pet health.

While crop species harbor many of the dietary requisites for basic human existence, numerous epidemiological studies suggest that plant-based foods contain varied and numerous compounds that can significantly impact human health beyond the maintenance of basic nutrition (Yochum et al., 2000). Such compounds are commonly referred to as phytonutrients or nutraceuticals. An example of particular interest to our research group is the broad class of antioxidants whose elevated consumption is associated with reductions in heart disease and decreased incidence of certain cancers (Rao and Agarwal, 2000). The development of genomics and bioinformatics tools has placed plant science on the threshold of developing traditional crops optimized for compounds that will not only meet the needs of basic nutrition, but will additionally reduce the incidence of degenerative disease and improve human health and resulting quality of life.
\end{abstract}

\section{EXAMPLES OF HEALTH-BASED MODIFICATION IN CROPS}

The elucidation of the carotenoid biosynthesis pathway in plants (reviewed in Cunningham and Gantt, 1998) has allowed researchers to contemplate engineering of this pathway in crop species (e.g., rice) not typically considered good sources of provitamin A carotenoids, yet which represent a significant source of dietary intake in countries where vitamin A deficiency is serious. So called "golden rice" resulted from engineering and delivery of transgenes that expressed three catalytic steps in beta-carotene biosynthesis for which rice endosperm is deficient (Ye et al., 2000). From a practical standpoint this effort is largely a proof of concept, as the resulting lines remain relatively poor sources of provitamin A. While significantly higher levels of carotenoid accumulation are needed to meet minimal nutrient requirements in areas where rice is the primary food source (and higher levels still would be needed for phytonutrient impact), this highly significant work demonstrated that a multistep biosynthetic pathway could be engineered in an important crop for promotion of human nutrition. Further efforts must now address means for elevating expression of these genes in addition to addressing questions of downstream caro-

Received for publication 29 Nov. 2001. Accepted for publication 30 Nov. 2001. This work was funded in part by the following: USDA-NRI (92-37300-7653, 95-37300-1575, 00-35300-9356), NSF(IBN-9604115, DBI-9872617), Zeneca Agrochemicals, Lipton Foods, Texas Agricultural Experiment Station, and USDA-ARS tenoid metabolism and sinks for carotenoid deposition and accumulation in rice.

Shintani and DellaPenna (1998) employed a genomics strategy to isolate a gene (gamma-tocopherol methyltransferase) responsible for the conversion of gamma-tocopherol to alpha-tocopherol (Vitamin E). Vitamin E cannot be synthesized by humans and must be acquired through the diet. A majority of tocopherol in many dietary sources of Vitamin E (e.g., soybean) is in the form of gamma-tocopherol, suggesting the potential for substantial elevation in net Vitamin E levels if conversion of gamma to alpha-tocopherol could be achieved. When overexpressed in Arabidopsis (another high gamma accumulator), gamma-tocopherol methyltransferase resulted in a substantial shift in the tocopherol pool to the Vitamin E form. This single gene manipulation represents one of the most promising near-term applications of biotechnology for improvement of human health, as levels of Vitamin $\mathrm{E}$ above dietary requirements have been implicated in reduced incidence of degenerative disease (Pryor, 2000).

A second and equally significant area of health-based crop modification is the use of plants as an efficient and affordable means of pharmaceutical delivery. Most significant in this regard are efforts toward development of plant-based vaccines (Walmsley and Arntzen, 2000). While not all antigens can be delivered successfully via ingestion, those that can often represent disease problems that are typically acquired via ingestion. While plant-based human vaccines are not yet commercially available, several experiments where expression of antigens in plants resulted in antibody production or immunity in test animals and/or humans have been reported. Examples include development of immune responses in humans to Norwalk virus antigen delivered via transgenic potato tubers (Tacket et al., 2000), and immunity to enterotoxin LT of $E$. coli in mice who have eaten potatoes expressing the LT antigen (Haq et al., 1995; Mason et al., 1998).

\section{TOMATO FRUIT AS A SYSTEM FOR DISCOVERY AND DELIVERY OF HEALTH BENEFITS}

The ripening of fleshy fruits represents a system of eukaryotic development unique to plants as well as an important component of agricultural production and human food consumption and nutrition. Greater understanding of the genetic and molecular basis of the ripening process will promote both our collective understanding of plant development and additionally yield tools useful for sustaining and improving: 1) agricultural productivity; 2) resulting fresh and processed fruit quality; and 3) human health and nutrition; while 4) minimizing impact on the environment necessary for production. Research in our laboratory focuses on tomato, with emphasis on gaining insights relative to genetic processes that impact the nutritional quality of fruits via both targeted modification of specific compounds and overall control and improvement of ripening quality.

Because it is diploid, has a modest-sized genome, and tolerates inbreeding, tomato is generally more amenable to genetic and molecular studies than other solanaceous species, many of which are polyploid (e.g., potato and tobacco) or have considerably larger genomes (e.g., pepper). As a result, a vast knowledge base has resulted for tomato genetics, cytogenetics, and taxonomy. In addition, numerous genetic and molecular tools are available for tomato research, such as a high-density molecular linkage map (Tanksley et al., 1992), introgression and substitution lines (Eshed and Zamir, 1994), Bacterial and Yeast Artificial Chromosome (BAC/YAC) in addition to cosmid and cDNA libraries, routine transformation, including binary-BAC (BIBAC) transformation of large inserts (Hamilton et al., 1999), and a set of strategically located transposon lines (Knapp et al., 1994; Osborne et al., 1991). In addition, more than 2000 mutants have been 
Table 1. Summary of tomato Expressed Sequence Tag (EST) sequencing and objectives. Tissues/libraries targeted for library construction as well as the actual number of ESTs generated from each library as of Dec. 2000. Sequencing and database construction is done by The Institute for Genome Research (TIGR).

\begin{tabular}{lrr}
\hline \hline & \# Completed & \\
cDNA Library & ESTs & Target \\
\hline Callus & 10,072 & 10,000 \\
Fruit (5 libraries 5d pre-anthesis_-overripe) & 33,371 & 30,000 \\
Pseudomonas susceptible and resistant plant & 11,379 & 10,000 \\
Mixed elicitors (of defense response) & 11,912 & 10,000 \\
Seed (germinating and dormant) & 5,040 & 9,000 \\
Roots (3 stages and mineral stress) & 12,811 & 12,000 \\
Trichomes & 5,636 & 5,000 \\
Flowers & 11,038 & 10,000 \\
Shoots & 1,348 & 10,000 \\
Selected clones (via screens) & & 5,000 \\
Mapped markers (3' and 5') & & 2,000 \\
Suspension culture (subtracted, various-inducers) & & 15,000 \\
Suspension culture (control) & & 5,000 \\
Pollen tubes (germinating) & 5,368 & 5,000 \\
Callus (agro-induced) & & 5,000 \\
Demethylated-genomic DNA & & 10,000 \\
\hline Total & $\mathbf{1 0 7 , 9 7 5}$ & $\mathbf{1 5 3 , 0 0 0}$ \\
\hline
\end{tabular}

characterized in tomato as well as more than 500 QTLs (Quantitative Trait Loci) controlling numerous aspects of fruit development yield and quality. A recently added tool to the repertoire of tomato and other plant science researchers is the National Science Foundation-sponsored development of a tomato EST (Expressed Sequence Tag or random cDNA clone) database. Twenty-five cDNA libraries from various tissues have been created, followed by partial (single-pass $5^{\prime}$ ) sequencing of 2,000 to 10,000 clones from each (Table 1). The database will be at or near completion by Fall 2001 and can be accessed prior to and following completion via the following web address (http://www.tigr.org/tdb/lgi/). The database includes $\approx 30,000$ sequences derived from fruit at various stages of development, and a query conducted following the completion of all fruit tissue sequencing indicated $\approx 1000$ non-redundant ESTs that are found exclusively in the subset of fruit libraries.

\section{GENETIC CONTROL OF FRUIT RIPENING}

The ripe phenotype is the summation of biochemical and physiological changes occurring at the final stages of fruit development, ultimately rendering the organ palatable and desirable to seed-dispersing animals and often valuable as an agricultural commodity. These changes, although varying greatly species to species, generally include modification of cell wall structure with resulting impact on ripe fruit texture, conversion of starch to sugars, increased susceptibility to opportunistic postharvest pathogens, alterations in pigmentation, and alteration of processes impacting levels of flavor and aroma constituents (Rhodes, 1980). A number of ripening attributes contribute to decreased shelf life and high input harvest, shipping and storage practices, particularly via changes in firmness, and the overall decrease in resistance to microbial infection associated with fruit ripening. Early harvest and controlled environment storage practices designed to counter these negative ripening consequences unfortunately also adversely impact fruit quality and flavor. Significant positive impact on human health could be achieved via development of genetic and cultural approaches that result in durable high-quality fruits, which in turn drive increased consumption.

In climacteric fruit such as tomato, ethylene clearly plays an important role in coordination of the ripening process (see Klee and Clark from this colloquium presentation). Further analysis of transgenic and mutant tomato lines inhibited in ethylene biosynthesis or perception demonstrates that climacteric ripening represents a combination of ethylene regulation and developmental control. Indeed, members of the gene family encoding the rate limiting activity in ethylene biosynthesis, ACC synthase, are in some instances initially induced during ripening by a developmental signal that remains to be defined, yet does not seem to be related to ethylene (Barry et al., 2000; Theologis et al., 1993). Analysis of expression of a number of additional ripeningrelated genes indicates that "developmental" or "non-ethylene mediated" regulation of a subset of ripening-related genes is evident in climacteric fruits. Examples in tomato include members of the ACO and ACS gene families (Barry et al., 2000; Blume and Grierson, 1997; Nakatsuka et al., 1998), the $N r$ ethylene receptor (Wilkinson et al., 1995), and E8 (Deikman et al., 1998). Additional evidence for nonethylene mediated ripening control comes from analysis of gene expression in a number of ripening-impaired mutants, such as rin (ripening-inhibitor) and nor (non-ripening), that fail to ripen in response to exogenous ethylene, yet display signs of ethylene sensitivity and signaling, including induction of some ethylene-regulated genes (Yen et al., 1995). Others and we have interpreted these results to indicate that additional regulatory constraints are placed on climacteric fruit maturation in addition to general ethylene biosynthesis and signaling. Such regulatory mechanisms could include fruit-specific regulation of certain subsets of ethylene-regulated genes or regulatory mechanisms that operate separate from and in addition to ethylene. Genes corresponding to both the rin and nor mutations have been recently cloned, and while unrelated at the level of DNA or protein sequence, both have features suggestive of roles in regulation of gene transcription (Giovannoni et al, unpublished). Availability of these ripening regulatory genes should allow analysis of steps in the ripening regulatory hierarchy preceding ethylene, and should additionally permit assessment of whether or not such genes represent regulatory mechanisms common to additional fruit species and possibly including non-climacteric fruits. From the standpoint of improved fruit quality, availability of such regulatory genes will provide additional genetic tools for regulation and modification of ripening parameters that may prove more efficient and with less negative consequences than is currently possible.

\section{HIGH-PIGMENT MUTATIONS}

The green to red color transition typical of ripening tomato fruit is due largely to the transition of chlorophyll-containing chloroplasts to carotenoid-rich chromoplasts. This occurs as chlorophyll and associated photosynthetic membranes are metabolized, and carotenoids, including $\beta$-carotene and lycopene, accumulate. While numerous tomato mutants altered in pigment accumulation have been reported (Table 2), few that result in net carotenoid accumulation have been identified. A combination of biochemical and genetic approaches has resulted in the isolation of a key gene, lycopene-epsilon-cyclase, responsible for the relative levels of $\beta$-carotene and lycopene in tomato fruit (Ronen et al., 1999). Discovery of this gene also led to elucidation

Table 2. Tomato mutants altered in carotenoid accumulation. Additional mutants exist but are much less well characterized in terms of carotenoids.

\begin{tabular}{llll}
\hline \hline Genotype & \multicolumn{1}{c}{ Activity } & \multicolumn{1}{c}{ Function } & \multicolumn{1}{c}{ Reference } \\
\hline rin; ripening-inhibitor & Transcription factor & Comprehensive ripening & Tigchellar et al., 1978 \\
nor; non-ripening & Transcription factor & Comprehensive ripening & Tigchellar et al., 1978 \\
Nr: Never-ripe & Ethylene receptor & Ethylene signaling & Wilkinson et al., 1995 \\
hp-2; high-pigment-2 & DET1 homolog & Light signaling & Mustilli et al., 1999 \\
cr; crimson & Lycopene cyclase & Carotenoid metabolism & Ronen et al., 1999, 2000 \\
B; Beta & Lycopene cyclase & Carotenoid metabolism & Ronen et al., 1999, 2000 \\
$r ;$ Phytoene Synthase & Phytoene synthase & Carotenoid metabolism & Fray and Grierson, 1993 \\
hp-1; high-pigment -1 & NA (not cloned) & Light signaling & Yen et al., 1997 \\
\hline
\end{tabular}

J. Vrebalov and J. Giovannoni et al., unpublished. 
of the molecular basis of the tomato $b$ (Beta) and $\operatorname{cr}$ (crimson) mutants that result in fruit preferentially accumulating either $\beta$-carotene or lycopene, depending on enhanced or reduced expression of the cyclase gene, respectively (Ronen et al., 2000).

Molecular regulation of the role of light in fruit ripening has been studied most thoroughly to date in tomato, and available evidence suggests that light has its greatest impact on pigmentation, with apparently little effect on additional ripening phenomena. Alba et al. (2000) demonstrated that tomato fruit placed in light-tight bags during development ripened in all normal aspects excepting greatly inhibited carotenoid accumulation.

A particularly interesting mutation from the standpoint of fruit carotenoid accumulation and the relationship of this aspect of ripening to light is the recessive $h p-1$ (high pigment-1) mutation. In contrast to most tomato carotenoid mutations, $h p-1$ results in increased accumulation of both lycopene and $\beta$-carotene during fruit development, in addition to elevated levels of chlorophyll in leaves and green fruit at all stages of development (Wann et al., 1985). We have additionally shown that $h p-1 / h p-1$ ripe fruit accumulate $\approx 20$ times the amount of the antioxidant flavonoid quercetin as compared to identically aged fruit from normal nearly isogenic lines. $h p-1$ fruit also preferentially accumulate sucrose at the expense of glucose and fructose. Consistent with a role in light signaling, we have also shown that mutation at the $h p-1$ locus results in several-fold amplification of the plastid genome (Yen et al., 1997). A mutation similar in phenotype to $h p-1$ and named $h p-2$ was described by Soressi (1975) and shown to be nonallelic with $h p-1$.

Important for understanding the underlying genetic basis of the $h p$ 1 mutation is the observation that $h p-1$ seedlings demonstrate an exaggerated photomorphogenic (de-etiolation) response (Peters et al., 1989). To summarize, $h p-1 / h p-1$ seedlings are characterized by inhibition of hypocotyl elongation and intense anthocyanin pigmentation relative to seedlings of the normal near isoline, with maximal phenotypic expression occurring in response to red light (Peters et al., 1992). Tomato seedling de-etiolation is a phytochrome (red light) response, which can be enhanced by blue light, suggesting that $h p-1$ may influence phytochrome and blue light receptor action or signaling. Overexpression of oat phytochrome A in tomato resulted in phenotypes similar to those observed in the $h p-1$ mutant, including increased carotenoid accumulation in ripe fruit (Boylan and Quail, 1989). Importantly, the $h p-1$ phenotype was repressed when associated with the phytochrome-deficient aurea (au) mutant, confirming the role of $h p-1$ in phytochrome responses. In addition, quantitation of phytochrome levels in normal and $h p-1 / h p-1$ seedlings indicates that the amplified phytochrome responses observed in the $h p-1$ mutant occur in the context of normal phytochrome concentration and stability, suggesting that the normal $H P-1$ gene product acts as a negative regulator of phytochrome signal transduction in tomato (Peters et al., 1992).

Arabidopsis is the most widely studied plant system for elucidating the genetic basis of light signal transduction. As a result, a large number of mutations have been identified and corresponding genes that represent various negative regulatory functions related to light signaling have been cloned (reviewed in Chemovitz and Deng, 1996). Such genes may be postulated to be similar in function to $\mathrm{Hp}$ - 1 . Indeed the tomato $h p-2$ mutation was recently shown to represent a tomato homologue of Arabidopsis DET1 (DE-ETIOLATED1) (Mustilli et al., 1999). This result confirmed the role of general light signaling in fruit pigment accumulation and suggests that a greater understanding of these processes may lead to successful efforts toward fruit quality and nutrient modification. Efforts toward isolation of the $h p-1$ locus via a positional cloning strategy are ongoing in our lab (Yen et al., 1997).

\section{THE PROMISE OF HEALTH-BASED GENOMICS}

Elucidation of the carotenoid biosynthesis pathway yielded important tools for modification of plant carotenoid levels. Nevertheless, while important pathways for biosynthesis of carotenoids and additional phytonutrients may be understood, regulatory hierarchies that control metabolite flux through said pathways are largely unknown. Development of comprehensive genomic and EST sequence databases, such as that currently being developed for tomato (Table 1), combined with technologies for large-scale expression profiling and data tracking, should allow for greater understanding of geneticregulatory networks underlying nutrient and health properties of plantbased foods. Resulting insights will lead to more efficient and targeted manipulations that will optimize health benefits with minimal adverse impact on quality.

\section{Literature Cited}

Alba, R., M.M. Cordonnier-Pratt, and L. Pratt. 2000. Fruit-localized phytochromes regulate lycopene accumulation independently of ethylene production in tomato. Plant Physiol. 123:363-70.

Barry, C.S., I. Llop-Tous, and D. Grierson. 2000. The regulation of 1aminocyclopropane-1-carboxylic acid synthase gene expression during the transition from system- 1 to system-2 ethylene synthesis in tomato. Plant Physiol. 123:979-86.

Blume, B. and D. Grierson. 1997. Expression of ACC oxidase promoter-GUS fusions in tomato and Nicotiana plumbaginifolia regulated by developmental and environmental stimuli. Plant J. 12:731-46.

Boylan, M.T. and P.H. Quail. 1989. Oat phytochrome is biologically active in transgenic tomatoes. Plant Cell 1:765-73.

Chamovitz, D.A. and X.W. Deng. 1996. Light signaling in plants. Crit. Rev. Plant Sci. 15:455-78.

Cunningham, F.X. and E. Gantt. 1998. Genes and enzymes of carotenoid biosynthesis in plants. Ann. Rev. Plant Physiol. Plant Mol. Biol. 49:557-583.

Deikman, J., R. Xu, M.L. Kneissl, J.A. Ciardi, K.N. Kim, and D. Pelah. 1998. Separation of cis elements responsive to ethylene, fruit development, and ripening in the $5^{\prime}$-flanking region of the ripening-related E8 gene. Plant Mol. Biol. 37:1001-11.

Eshed, Y. and D. Zamir. 1994. A genomic library of Lycopersicon pennellii in L. esculentum: A tool for fine mapping of genes. Euphytica 79:175-9.

Fray, R.G. and D. Grierson. 1993. Identification and genetic analysis of normal and mutant phytoene synthase genes of tomato by sequencing, complementation, and co-suppression. Plant Mol. Biol. 22:589-602.

Hamilton, C.M., A. Frary, Y. Xu, S. Tanksley, and H.B. Zhang. 1999. Construction of tomato genomic DNA libraries in a binary-BAC (BIBAC) vector. Plant J. 18:223-9.

Haq, T.A., H.S. Mason, J.D. Clements, and C.J. Arntzen. 1995. Oral immunization with a recombinant bacterial antigen produced in transgenic plants. Science 268:714-716.

Knapp, S, Y. Larondelle, M. Rossberg, D. Furtek, and K. Terres. 1994. Transgenic tomato lines containing Ds elements at defined genomic positions as tools for targeted transposon tagging. Mol. \& Gen. Genet. 243:666-73.

Mason, H.S., T.A. Haq, J.D. Clements, and C.J. Arntzen. 1998. Edible vaccine protects mice against Escherichia coli heat-labile enterotoxin (LT): Potatoes expressing a synthetic LT-B gene. Vaccine 16:1336-1343.

Mustilli, A.C., F. Fenzi, R. Ciliento, F. Alfano, and C. Bowler. 1999. Phenotype of the tomato high pigment-2 mutant is caused by a mutation in the tomato homolog of DEETIOLATED1. Plant Cell 11:145-57.

Nakatsuka, A., S. Murachi, H. Okunishi, S. Shiomi, R. Nakano, Y. Kubo, and I. Akitsugu. 1998. Differential expression and internal feedback regulation of 1-aminocyclopropane-1-caboxylate synthase, of 1-aminocyclopropane1-caboxylate oxidase, and ethylene receptor genes in tomato during development and ripening. Plant Physiol. 118:1295-305.

Osborne, B., C. Corr, J. Prince, R. Hehl, S. Tanksley, S. McCormick, and B. Baker. 1991. Ac transposition from a T-DNA can generate linked and unlinked clusters of insertions in the tomato genome. Genetics 129:833-44.

Peters, J.L., M.L. Schreuder, S.W. Verduin, and R.E. Kendrick. 1992. Physiological characterization of a high-pigment mutant of tomato. Photochem. and Photobiol. 56:75-82.

Peters, J.L., A. van Tuinen, P. Adamse, R.E. Kendrick, and M. Koornneef. 1989. High pigment mutants of tomato exhibit high sensitivity for phytochrome action. J. Plant Physiol. 134:661-6.

Pryor, W.A. 2000. Vitamin E and heart disease: Basic science to clinical intervention trials. Free Radical Biol. and Med. 28:141-164.

Rao, A.V. and S. Agarwal. 2000. Role of antioxidant lycopene in cancer and heart disease. J. Amer. Coll. Nut. 19:563-569.

Rhodes, M. 1980. The maturation and ripening of fruits, p. 157-205. In: K.V. Thimann (ed.). Senescence in plants. CRC Press, Boca Raton, Fla.

Ronen, G., M. Cohen , D. Zamir, and J. Hirschberg. 1999. Regulation of carotenoid biosynthesis during tomato fruit development: Expression of the gene for lycopene epsilon-cyclase is down-regulated during ripening and is elevated in the mutant Delta. Plant J. 17:341-51.

Ronen, G., G.L. Carmel, D. Zamir, and J. Hirschberg. 2000. An alternative pathway to beta-carotene formation in plant chromoplasts discovered by map-based cloning of Beta and old-gold color mutations in tomato. PNAS USA 97:11102-7.

Shintani, D. and D. Dellapenna. 1998. Elevating the vitamin E content of plants through metabolic engineering. Science 282:2098-2100. 
Soressi, G.P. 1975. New spontaneous or chemically-induced fruit ripening tomato mutants. Tomato. Genet. Coop. Rep. 25:21-2.

Tacket, C.O., H.S. Mason, G. Losonsky, M.K. Estes, M.M. Levine, and C.J. Arntzen. 2000. Human immune responses to a novel Norwalk virus vaccine delivered in transgenic potatoes. J. Infec. Dis. 182:302-305.

Tanksley S., M. Ganal, J. Prince, C. deVincente, M. Bonierbale, P. Broun, T. Fulton, J. Giovannoni, S. Grandillo, G. Martin, R. Messeguer, J. Miller, L. Miller, A. Paterson, O. Pineda, M. Roder, R. Wing, W. Wu, and N. Young. 1992. High density molecular maps of the tomato and potato genomes. Genetics 132:1141-1160.

Theologis, A., P.W. Oeller, L.M. Wong, W.H. Rottmann, and D.M. Gantz. 1993. Use of a tomato mutant constructed with reverse genetics to study fruit ripening, a complex developmental process. Dev. Gen. 14:282-95.

Tigchelaar, E., W. McGlasson, and R. Buescher. 1978. Genetic regulation of tomato fruit ripening. HortScience 13:508-13.

Walmsley, A.M. and C.J. Arntzen. 2000. Plants for delivery of edible vaccines. Current Opinion in Biotech. 11:126-129.

Wann, E.V., E.L. Jourdain, R. Pressey, and B.G. Lyon. 1985. Effect of mutant genotypes $h p o g^{c}$ and $d g \mathrm{og}^{c}$ on tomato fruit quality. J. Amer. Soc. Hort. Sci. 110:212-215.

Wilkinson, J., M. Lanahan, H. Yen, J. Giovannoni, and H. J. Klee. 1995. An ethylene-inducible component of signal transduction encoded by Neverripe. Science 270:1807-9.

Ye, X., B.S. Al, A. Kloti, J. Zhang, P. Lucca, P. Beyer, and I. Potrykus. 2000. Engineering the provitamin A (beta-carotene) biosynthetic pathway into (carotenoid-free) rice endosperm. Science 287:303-305.

Yen, H., S. Lee, S. Tanksley, M. Lanahan, H.J. Klee, and J.J. Giovannoni. 1995. The tomato Never-ripe locus regulates ethylene-inducible gene expression and is linked to a homologue of the Arabidopsis ETRl gene. Plant Physiol. 107:1343-53.

Yen, H., B. Shelton, L. Howard, J. Vrebalov, and J.J. Giovannoni. 1997. The tomato high pigment $(h p)$ locus maps to chromosome 2 and influences plastome copy number and fruit quality. Theor. Appl. Gen. 95:1069-79.

Yochum, L.A., A.R. Folsom, and L.H. Kushi. 2000. Intake of antioxidant vitamins and risk of death from stroke in postmenopausal women. Amer. J. Clin. Nut. 72:476-483.

\title{
Molecular Biology of Host-Pathogen Interactions in Harvested Horticultural Crops
}

\author{
T.G. McCollum \\ USDA, ARS, USHRL, Ft. Pierce, FL 34945
}

\begin{abstract}
Decay caused by fungal plant pathogens can lead to considerable postharvest losses. Estimates of postharvest losses due to decay vary widely with commodity, production area, and season, but it is well known that these losses can be significant (Burchill and Maude, 1986; Eckert 1975; Pathak, 1997). Fungicides, applied both pre- and postharvest, along with environmental management are utilized to minimize postharvest decay. About 50 million pounds of fungicides are applied to fruits and vegetables annually, and it is generally accepted that production and marketing of these products would not be possible without the use of fungicides (Ragsdale and Sisler, 1994). However, public concern over the freedom of fresh fruits and vegetables from pesticides, increasing regulatory constraints for the registration of pesticides, and the development of resistance to fungicides indicate that the availability and use of fungicides is certain to diminish (Ragsdale and Sisler, 1994; Sutton, 1996). The potential loss of fungicides necessitates that alternative strategies must be developed for reducing losses due to postharvest decay.
\end{abstract}

Fortunately, freedom from decay is the norm, rather than the exception. Of the $>100,000$ species of fungi, $<10 \%$ are plant pathogens and $<100$ species of fungi are responsible for the majority of postharvest diseases (Eckert and Ratnayake, 1983). Although some plant pathogens, such as Botrytis cinerea and Colletotrichum sp., have wide host ranges, most fungal species responsible for postharvest decay have a limited number of hosts from related plant species. For example, Penicillium digitatum causes green mold on citrus fruits, but does not cause disease in apples and pears. Penicillium expansum attacks apples and pears, but not citrus fruits. These are examples of "non-host resistance." Non-host resistance is the consequence of: 1) inability of the fungus to recognize and infect the potential host or 2) the ability of the plant to rapidly and successfully activate defense responses (Kombrink and Somssich, 1995).

Unfortunately, when fruits and vegetable tissues are harvested, their resistance to decay is compromised. Injuries incurred during harvesting and handling operations provide ports of entry for pathogens that cannot penetrate unaltered tissues directly. Harvesting initiates senescence even in physiologically immature organs, and ripening is a specialized form of senescence unique to fruits. Consequences of senescence include both the loss of preformed barriers to pathogens as well as a decrease in the ability to mount an active defense. As a result, the incidence of decay increases (Labavitch, 1998). Many

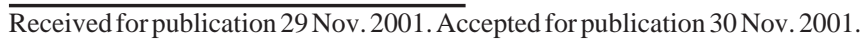

postharvest diseases result from infections that occur early in the life of the fruit, but which remain quiescent until ripening commences (Eckert and Ranayake, 1983; Prusky, 1996). Understanding how natural defense mechanisms are regulated and how to maintain them in harvested products may provide the basis for new strategies to reduce postharvest losses caused by pathogens.

Considerable progress has been made in understanding the interaction between pathogen and host, as evidenced by numerous recent reviews on various aspects of the subject (Dixon and Lamb, 1990; Dixon et al., 1994; Hahn, 1996; Keen, 1992; Knogge, 1996). For the purpose of this review I have chosen to limit my discussion primarily to responses induced in the pathogen by the host and in the host by the pathogen. In addition, in keeping with the theme of the colloquium, I have chosen to focus on studies conducted specifically with harvested horticultural crops in order to illustrate concepts regarding molecular aspects of the host-pathogen interaction.

\section{THE PATHOGEN}

The first step in the infection process is recognition of the host by the pathogen, and this is no doubt regulated by signals arising from the host. Various chemical signals from the host have been identified as being stimulatory to germination of the pathogen. Volatiles emitted from wounded oranges stimulate the germination of $P$. digitatum conidia (Eckert and Ratnayake, 1994). Ethylene has also been suggested as a host-generated signal that stimulates fungal germination. Flaishman and Kolattukudy (1994) reported that ethylene at concentrations $\leq 1 \mathrm{ppm}$ caused germination, branching of the germ tube, and formation of up to six appressoria from single spores of Colletotrichum gloeosporioides. Colletotrichum gloeosporioides formed multiple appressoria on wild-type tomato fruit, but not on transgenic tomatoes that lacked the capacity to produce ethylene. Treatment of inoculated transgenic fruit with ethylene resulted in infection and lesion development (Flaishman and Kolattukudy, 1994). Sensitivity of Diplodia natalensis to scoporone, an antifungal compound in citrus rind, was reduced and fungal growth rate was increased when the fungus was grown in the presence of ethylene (Brown and Lee, 1993).

Compounds present in the cuticle have also been shown to stimulate the germination and germ tube growth of pathogens. Surface waxes from avocado stimulate germination and formation of appressoria from the spores of $C$. gloeosporioides. Reciprocally, waxes from nonhost species do not induce appressoria. Furthermore, avocado wax 